\title{
Inserção de egressos de Odontologia do Tocantins no mercado de trabalho
}

Bruno Arlindo de Oliveira Costa*; Cintia Ferreira Gonçalves**; Luciane Zanin***; Flávia Martão Flório****

\footnotetext{
* Aluno de Pós-graduação, Departamento de Saúde Coletiva, Faculdade de Odontologia e Centro de Pesquisas Odontológicas São Leopoldo Mandic, Campinas, SP.

** Professora de Odontologia para Pacientes com Necessidades Especiais e Odontologia Hospitalar, Faculdade Antônio Carlos, Porto Nacional, TO.

Professora no Departamento de Saúde Coletiva, Faculdade de Odontologia e Centro de Pesquisas Odontológicas São Leopoldo Mandic, Campinas, SP Professora no Departamento de Saúde Coletiva, Faculdade de Odontologia e Centro de Pesquisas Odontológicas São Leopoldo Mandic, Campinas, SP.
}

\section{RESUMO}

O objetivo do presente trabalho foi investigar a inserção e a perspectiva profissional de egressos de Odontologia no mercado de trabalho. Trata-se de um estudo observacional, transversal e descritivo, realizado por meio de um questionário eletrônico aplicado aos 298 egressos de um curso de Odontologia do Tocantins, distribuídos em 14 turmas (2008 a 2015). A taxa de resposta foi de $61,41 \%(n=183)$. Após análise dos dados (Teste de Quiquadrado/Exato de Fisher; nível de significância de 5\%) verificou-se que $65,6 \%$ dos respondentes eram do sexo feminino, com idade média de $30( \pm 6,6)$ anos. O tempo médio de formado foi de 3,7 $( \pm 2,2)$ anos. Dentre os egressos, $37,8 \%$ trabalhavam em serviço público, sendo que $17,5 \%$ o faziam exclusivamente. A maioria deles trabalhava no estado do Tocantins $(65,0 \%)$, sendo que $62,8 \%$ estava cursando ou cursou pósgraduação, sendo Ortodontia (17,5\%),
Endodontia (14,8\%), Odontopediatria (7,6\%); a área de Saúde Coletiva apareceu para 3,8\% dos respondentes. A renda mensal da maioria $(46,4 \%)$ foi de até 5 salários mínimos $(\mathrm{R} \$ 3$ $640,00)$ e $49,8 \%$ dos respondentes mostraramse satisfeitos com o rendimento mensal. A inserção imediata no mercado de trabalho foi relatada por $72,7 \%$ dos egressos, sendo que $6 \%$ não exerciam a profissão naquele momento e destes, $2,7 \%$ nunca exerceram. Dentre as disciplinas consideradas mais significativas na graduação apareceram Cirurgia Oral (26,8\%), Endodontia (21,3\%), Dentística $(14,2 \%)$, Odontopediatria $(11,5 \%)$, Prótese $(9,3 \%)$ e Saúde Coletiva $(4,9 \%)$. Os resultados encontrados permitem concluir que a inserção dos egressos no mercado de trabalho foi rápida e a maioria se encontrava satisfeita com os rendimentos naquele momento.

Descritores: Mercado de Trabalho. Recursos Humanos em Odontologia. Formação de Recursos Humanos. 


\section{INTRODUÇÃO}

O curso de Odontologia foi instituído no Brasil em 1884, e desde então ocorreram consideráveis modificações nos cursos oferecidos por Instituições de Ensino Superior (IES) privadas, públicas, federais e municipais no país ${ }^{1}$.

Entre os anos 1980 e 1990 a Odontologia era uma das práticas de saúde mais elitizadas do Brasil $^{2}$. A inserção de cirurgiões-dentistas no setor público se limitava a precários serviços escolares e abomináveis rotinas de extração de dentes da população mais pobre ${ }^{3}$. As lutas pela democracia e pela extensão da cidadania, as Conferências de Saúde e a construção do Sistema Único de Saúde (SUS), previsto na Constituição Federal de 1988, impulsionaram mudanças nessas práticas e nas concepções sobre o quê, e como se deveria fazer a Odontologia brasileira, estabelecendo-se como princípios a integralidade da atenção à saúde, com ênfase nos aspectos preventivos, e uma atuação profissional dirigida à realidade sócio-epidemiológica da população do País ${ }^{4}$.

De acordo com dados do Conselho Federal de Odontologia (CFO) $(2015)^{5}$, o Brasil é atualmente o país com maior número de cirurgiões-dentistas do mundo e mesmo com esta quantidade de profissionais, a odontologia vivencia uma importante desigualdade na distribuição destes profissionais no território brasileiro ${ }^{6}$. Em sete Estados do Norte e Nordeste - Acre, Amapá, Pará, Piauí, Bahia, Ceará e Maranhão - existe, em média, um dentista para cada 1,8 mil habitantes. Já as Regiões Sul e Sudeste há uma concentração de quase 200 mil cirurgiõesdentistas, o que corresponde a mais de $70 \%$ de todos os profissionais existentes no Brasil ${ }^{7}$. Nessa linha de raciocínio, enquanto no estado de São Paulo a proporção de cirurgiãodentista: habitante é de 1:556 ${ }^{8}$, no Tocantins, essa proporção aumenta para 1:1077 habitantes ${ }^{9}$.

A Política Nacional de Saúde Bucal $(2004)^{10}$ - Brasil Sorridente - tem como objetivo garantir ações de promoção, prevenção e recuperação da saúde bucal para toda a população brasileira. Para isso, foram necessárias uma série de ações para ampliação do acesso ao tratamento odontológico por meio do SUS, levando-o a se tornar um grande empregador para os cirurgiões-dentistas: a atenção básica em saúde bucal está presente em 90\% (5.013) dos municípios brasileiros, contando com a presença de $30 \%$ dos dentistas do Brasil (64.826 profissionais), atingindo mais de 81 milhões de brasileiros ${ }^{6}$.

Vale ressaltar que as Diretrizes Curriculares Nacionais $(\mathrm{DCN})^{11}$ não impõem um caráter único para os diversos cursos distribuídos no Brasil, mas definem uma base formadora sólida, que deve se dispor a ser constantemente complementada pelas instituições de ensino superior (IES), com suas diferentes experiências, realidades e regionalidades ${ }^{12}$. A efetiva interação entre os serviços de saúde do SUS e a formação dos profissionais de saúde tem como objetivo capacitar os futuros profissionais às necessidades sociais da população ${ }^{3}$. As IES têm como grande desafio formar profissionais na área de saúde para exercerem sua atividade atendendo ao SUS e ao mercado de trabalho privado. A incorporação deste novo modelo capacita o profissional a ter uma percepção mais abrangente, dinâmica, complementar e integrada, tornando-o apto a romper com a arraigada visão de trabalho linear e fragmentada ${ }^{12}$.

A questão da formação de profissionais de saúde envolve diretamente as oportunidades advindas do mercado de trabalho, o perfil profissional e a satisfação das demandas populacionais. Assim, a articulação entre as políticas de educação e de saúde é fundamental para que as transformações sejam possíveis ${ }^{13}$. Entretanto, diante da problemática da formação de profissionais de saúde bucal, especialmente dos cirurgiões-dentistas, por esta ser caracterizada por uma abordagem biologicista, medicalizante, centrada e com forte caráter flexneriano, existe uma forte tendência a mudanças, impulsionadas pelo discurso de 
reorganização dos modelos de atenção e das práticas de saúde, sobretudo pelas políticas que configuram o SUS ${ }^{14}$.

Dentre as motivações que levam esses estudantes à escolha do curso podemos citar a, relação com a visão que os mesmos apresentam da Odontologia, do exemplo de profissionais, do status social elitista, das possibilidades de atuação no mercado de trabalho e da necessidade ou não de comprometimento social enquanto profissionais de saúde ${ }^{15}$.

O papel da universidade é preparar um profissional crítico e reflexivo, aplicando técnicas pedagógicas que facilitem sua inserção no mercado de trabalho como profissional ético e responsável. No entanto, estudos constataram que a formação do acadêmico de graduação em Odontologia caracteriza-se pelo individualismo com ânsia de lucros, alienamento da realidade, tendência curativista e desprezo ao serviço público odontológico, tendo em vista diretrizes curriculares, as quais não contemplam um efetivo processo de ensino-aprendizagem ${ }^{16}$. A real mudança no perfil de egressos profissionais não se dá somente com mudanças curriculares, mas, sim, com novas práticas de formação em saúde ${ }^{17}$. Uma maior aproximação da educação e da saúde com a realidade social do seu meio e uma participação articulada e efetiva minimizaria a iniquidade proporcionada pela Odontologia tecnicista e elitista predominante no país ${ }^{18}$.

Tendo em vista que os egressos de Odontologia se deparam com dificuldades de inserção no mercado de trabalho atual e pela grande necessidade de profissionais no sistema público em todos os municípios do interior do país, o objetivo do presente trabalho foi investigar a inserção $e$ a perspectiva profissional de egressos de Odontologia do Tocantins no mercado de trabalho

\section{METODOLOGIA}

Este estudo observacional transversal descritivo foi conduzido de acordo com os preceitos determinados pela Resolução 422 de 12/2012 do Conselho Nacional de Saúde do Ministério da Saúde, aprovado pelo Comitê de Ética em Pesquisa da Faculdade de Odontologia e Centro de Pesquisa São Leopoldo Mandic (parecer 1.239.101).

O curso de Odontologia do Instituto Tocantinense Presidente Antônio Carlos, Porto Nacional do Tocantins (ITPACPORTO) formou a sua primeira turma em 2008 e em 2015 completou 12 turmas formadas, resultando em 298 (duzentos e noventa e oito) egressos. Os endereços eletrônicos dos egressos foram obtidos na secretaria de graduação e por mídia social (Facebook). Foram excluídos da pesquisa os egressos que não puderem ser contatados (17) e que não responderam aos e-mails convite e às demais formas de contato estabelecidas $(\mathrm{n}=115)$.

O questionário utilizado neste estudo foi desenvolvido com base no estudo de Freire et al. $(2011)^{9}$ e no instrumento utilizado pela Comissão Própria de Avaliação da Faculdade de Odontologia São Leopoldo Mandic, direcionado ao egresso. Foi realizado o testereteste do questionário em uma amostra de conveniência e verificou-se que a concordância média foi de $74 \%$. O instrumento foi composto por 22 questões, sendo 9 subjetivas e 13 objetivas explorando o perfil socioeconômico e demográfico, com informações relativas ao tempo de formação e responsável pelo custeio da graduação; a atuação no mercado de trabalho, o setor de atividade do trabalho e mudança do Estado de origem para a atuação profissional atual; tempo para inserção no mercado de trabalho e grau de satisfação com a remuneração; conceitualização do curso e da faculdade, com informações relativas às disciplinas favoritas e à motivação para escolha da instituição.

$\mathrm{O}$ instrumento foi enviado aos egressos via endereço eletrônico, contendo um link para acesso ao questionário. Quinze dias após o envio, foi realizado um novo contato com o intuito de sanar dúvidas ou explicar sobre a pesquisa, confirmando o 
recebimento deste questionário, e solicitando a resposta e reenvio.

Os resultados foram apurados e quantificados em frequências absolutas e relativas, visando caracterizar as variáveis pesquisadas. Os dados foram analisados por tabelas de distribuição de frequências. Os testes de Qui-quadrado e Exato de Fisher foram utilizados. Todas as análises foram realizadas no programa $\mathrm{R}^{*}$ (Universidade de Auckland, Nova Zelândia), considerando o nível de significância de 5\%.

\section{RESULTADOS}

Do total de egressos, houve predominância no gênero feminino, com $65,6 \%$ da amostra. A idade média dos voluntários foi de $30( \pm 6,6)$ anos (mínimo de 22 e máximo de 66 anos). O tempo médio de formado foi de $3,7( \pm 2,2)$ anos, com tempo mínimo de 1 ano e máximo de 11 anos; 60,1 $\%$ dos egressos cursou a faculdade com recursos financeiros familiares. A maioria da amostra encontrava-se com idade entre 18 e 35 anos, com tempo de formado de até 3 (três) anos.

A tabela 1 apresenta dados relacionados ao perfil do egresso, percepção sobre a formação e vivência quanto ao mercado de trabalho. Vale destacar que $37,7 \%$ dos egressos não retornaram para seu estado de origem após a graduação. Dentre os egressos, $62,8 \%$ possuía ou estava cursando pós-graduação, sendo Ortodontia $(17,5 \%)$, Endodontia $(14,8 \%)$ e Odontopediatria $(7,6 \%)$ as áreas com maior predominância. Outro dado relevante, foi que $93,4 \%$ consideraram que os conhecimentos adquiridos durante a graduação de forma positiva para a atuação profissional.

A maioria dos entrevistados apresentou uma renda mensal de até 10 salários mínimos, sendo que do total da amostra 46,4\% recebiam até 5 salários mínimos. Quando questionados sobre o nível de satisfação com relação à renda mensal, $66,2 \%$ da amostra a classificou como regular ou boa.
Em relação ao quanto o egresso se sentia preparado para entrar no mercado de trabalho, em uma escala de 0 (totalmente despreparado) a 10 (totalmente preparado), a grande maioria $(76,0 \%)$ atribuiu nota maior que 5.

Em relação ao tempo para inserção no mercado de trabalho, 72,7\% dos egressos relataram que foi imediata e apenas $2,7 \%$ da amostra nunca exerceu a profissão.

Na tabela 2 nota-se que a maioria dos respondentes trabalhava no setor privado e apenas $17,5 \%$ tiveram o setor público como o único vínculo trabalhista. Pode-se notar que a maioria dos egressos trabalhava nos Estados de Tocantins $(65,0 \%)$ e Pará $(13,7 \%)$.

Na tabela 3 apresenta-se as disciplinas que mais despertaram interesse durante o curso de graduação e situação quanto ao exercício profissional. Do total da amostra, $6,0 \%$ não estavam exercendo a Odontologia naquele momento, dentre os quais $2,7 \%$ nunca a exerceram. Dentre os motivos pelos quais o egresso não exercia a profissão, foram citados mudança de área profissional, frustração, desemprego, gravidez e falta de tempo para cuidar de filhos.

\section{DISCUSSÃO}

A caracterização dos egressos do FAPAC/ITPAC-PORTO mostrou que a maioria deles era mulheres, de origem tocantinense, solteiras, jovens e sem filhos. A maior presença de egressos do gênero feminino vem de encontro com o processo de feminilização da profissão odontológica, notado desde a década de 1990 e também verificada em outros estudos no Brasil ${ }^{16,20-28}$.

Nos trabalhos que corroboram com o presente estudo, não foram abordadas considerações sobre o custeio da graduação, sendo verificado neste que $60 \%$ dos egressos tiveram sua graduação custeada por rendimentos familiares, mostrando que o nível econômico dos acadêmicos pode ser considerado bastante privilegiado quando considerada a renda familiar mensal brasileira ${ }^{4}$. 
Tabela 1. Perfil do egresso do curso de Odontologia FAPAC/ITPAC-PORTO.

\begin{tabular}{|c|c|c|c|}
\hline Variável & Categoria & $\mathbf{n}$ & $\%$ \\
\hline \multirow{4}{*}{ Mudança de local } & Cidade & 35 & 19,1 \\
\hline & Estado & 69 & 37,7 \\
\hline & Não se mudou & 73 & 39,9 \\
\hline & Não respondeu & 6 & 3,3 \\
\hline \multirow[t]{2}{*}{ Pós-graduação } & Não & 68 & 37,2 \\
\hline & Sim & 115 & 62,8 \\
\hline \multirow[t]{15}{*}{ Qual curso? } & Cirurgia e Traumatologia Bucomaxilofaciais & 2 & 1,1 \\
\hline & Dentística & 3 & 1,6 \\
\hline & Disfunção Temporomadibular & 1 & 0,6 \\
\hline & Endodontia & 27 & 14,8 \\
\hline & Pacientes Especiais & 1 & 0,6 \\
\hline & Odontologia Hospitalar & 1 & 0,6 \\
\hline & Implantodontia & 8 & 4,4 \\
\hline & Odontologia Legal & 2 & 1,1 \\
\hline & Ortodontia & 32 & 17,5 \\
\hline & Odontopediatria & 14 & 7,6 \\
\hline & Prótese & 5 & 2,7 \\
\hline & Radiologia & 3 & 1,6 \\
\hline & Saúde da família & 4 & 2,2 \\
\hline & Saúde Pública & 3 & 1,6 \\
\hline & Não respondido & 9 & 4,9 \\
\hline \multirow[t]{2}{*}{ Como classificaria o conhecimento } & $<=5$ & 12 & 6,6 \\
\hline & $>5$ & 171 & 93,4 \\
\hline \multirow[t]{4}{*}{ Inserção no mercado } & Imediata & 133 & 72,7 \\
\hline & Menos de 1 ano & 38 & 20,8 \\
\hline & Mais de 1 ano & 7 & 3,8 \\
\hline & Não está inserido & 5 & 2,7 \\
\hline
\end{tabular}




\begin{tabular}{|c|c|c|c|}
\hline \multirow{3}{*}{ Preparação } & & & (continuação) \\
\hline & $<=5$ & 44 & 24,0 \\
\hline & $>5$ & 139 & 76,0 \\
\hline \multirow[t]{5}{*}{ Média Salarial } & Acima de 20 SM & 2 & 1,1 \\
\hline & Entre 11 e $20 \mathrm{SM}$ & 13 & 7,1 \\
\hline & Entre 5 e $10 \mathrm{SM}$ & 72 & 39,3 \\
\hline & Até $5 \mathrm{SM}$ & 85 & 46,4 \\
\hline & Não respondido & 11 & 6,0 \\
\hline \multirow[t]{6}{*}{ Satisfação com o salário } & Excelente & 12 & 6,6 \\
\hline & Muito bom & 15 & 8,2 \\
\hline & Bom & 64 & 35,0 \\
\hline & Regular & 57 & 31,2 \\
\hline & Ruim & 24 & 13,1 \\
\hline & Não respondido & 11 & 6,0 \\
\hline \multirow[t]{4}{*}{ Clientela } & População de baixa renda & 65 & 35,5 \\
\hline & População de renda média & 103 & 56,3 \\
\hline & População de renda alta & 2 & 1,1 \\
\hline & Não respondido & 13 & 7,1 \\
\hline
\end{tabular}

SM: Salários mínimos 
Tabela 2. Distribuição de frequências da atividade profissional atual em função do Estado de atuação.

\section{Atividade profissional}

\begin{tabular}{|c|c|c|c|c|c|c|c|c|}
\hline Estado Atual & 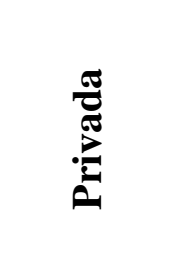 & 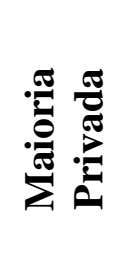 & 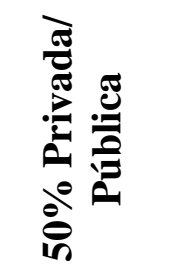 & 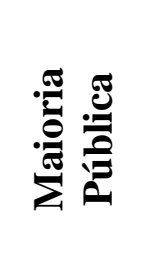 & 胥 & 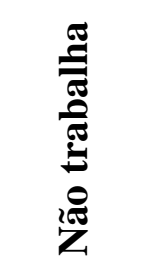 & 疍 & \\
\hline & & & & $\mathrm{N}\left(\%^{\$}\right)$ & & & & $\mathrm{N}\left(\%^{\&}\right)$ \\
\hline $\mathbf{B A}$ & $6(60,0)$ & $0(0,0)$ & $2(20,0)$ & $0(0,0)$ & $1(10,0)$ & $1(10,0)$ & $0(0,0)$ & $10(5,5)$ \\
\hline $\mathrm{CE}$ & $1(100,0)$ & $0(0,0)$ & $0(0,0)$ & $0(0,0)$ & $0(0,0)$ & $0(0,0)$ & $0(0,0)$ & $1(0,5)$ \\
\hline GO & $7(58,3)$ & $0(0,0)$ & $2(16,7)$ & $1(8,3)$ & $2(16,7)$ & $0(0,0)$ & $0(0,0)$ & $12(6,6)$ \\
\hline MA & $3(75,0)$ & $0(0,0)$ & $0(0,0)$ & $1(25,0)$ & $0(0,0)$ & $0(0,0)$ & $0(0,0)$ & $4(2,2)$ \\
\hline MT & $2(66,7)$ & $0(0,0)$ & $0(0,0)$ & $0(0,0)$ & $1(33,3)$ & $0(0,0)$ & $0(0,0)$ & $3(1,6)$ \\
\hline PA & $18(72,0)$ & $1(4,0)$ & $4(16,0)$ & $0(0,0)$ & $0(0,0)$ & $2(8,0)$ & $0(0,0)$ & $25(13,7)$ \\
\hline PE & $1(50,0)$ & $0(0,0)$ & $1(50,0)$ & $0(0,0)$ & $0(0,0)$ & $0(0,0)$ & $0(0,0)$ & $2(1,1)$ \\
\hline PI & $0(0,0)$ & $0(0,0)$ & $0(0,0)$ & $0(0,0)$ & $2(1,0)$ & $0(0,0)$ & $0(0,0)$ & $2(1,1)$ \\
\hline PR & $2(66,7)$ & $0(0,0)$ & $0(0,0)$ & $0(0,0)$ & $0(0,0)$ & $1(33,3)$ & $0(0,0)$ & $3(1,6)$ \\
\hline $\mathbf{R S}$ & $1(50,0)$ & $0(0,0)$ & $0(0,0)$ & $0(0,0)$ & $0(0,0)$ & $1(50,0)$ & $0(0,0)$ & $2(1,1)$ \\
\hline TO & $55(46,2)$ & $6(5,0)$ & $16(13,4)$ & $10(8,4)$ & $26(21,8)$ & $5(4,2)$ & $1(0,8)$ & $119(65,0)$ \\
\hline Total & $96(52,4)$ & $7(3,8)$ & $25(13,7)$ & $12(6,6)$ & $32(17,5)$ & $10(5,5)$ & $1(0,4)$ & $183(100,0)$ \\
\hline
\end{tabular}

${ }^{\$}$ Porcentagem nas linhas; ${ }^{\&}$ Porcentagens na coluna 
Tabela 3. Distribuição de frequências relacionadas à predileção por disciplinas durante o curso, exercício atual da profissão e razões para desistência.

\begin{tabular}{|c|c|c|c|}
\hline Variável & Categoria & $\mathbf{N}$ & $\%$ \\
\hline \multirow{14}{*}{ Disciplinas favoritas } & Anatomia/cirurgia & 1 & 0,6 \\
\hline & Cirurgia oral & 49 & 26,8 \\
\hline & Dentística & 26 & 14,2 \\
\hline & Endodontia & 39 & 21,3 \\
\hline & Implantodontia & 2 & 1,1 \\
\hline & Laser & 1 & 0,6 \\
\hline & Odontopediatria & 21 & 11,5 \\
\hline & Pacientes Especiais & 1 & 0,6 \\
\hline & Ortodontia & 8 & 4,4 \\
\hline & Periodontia & 5 & 2,7 \\
\hline & Prótese & 17 & 9,3 \\
\hline & Radiologia & 1 & 0,6 \\
\hline & Saúde coletiva & 9 & 4,9 \\
\hline & Não respondido & 3 & 1,6 \\
\hline \multirow[t]{2}{*}{ Atualmente exerce a Odontologia? } & Sim & 172 & 94,0 \\
\hline & Não & 11 & 6,0 \\
\hline \multirow[t]{7}{*}{ Se não, por quê? } & Desemprego & 1 & 9,1 \\
\hline & Filhos & 1 & 9,1 \\
\hline & Frustração & 1 & 9,1 \\
\hline & Gravidez & 1 & 9,1 \\
\hline & Mudou de área & 2 & 18,2 \\
\hline & Não quer & 1 & 9,1 \\
\hline & Não respondido & 4 & 2,2 \\
\hline
\end{tabular}


A implantação de uma nova Diretriz Curricular Nacional (DCN) deve ser seguida pelas IES em todo o pais, contemplando a adequação ao perfil profissional que vá ao encontro daquilo que é desejável pelo SUS. Neste estudo, observa-se também que a inserção no setor público foi menor do que a verificada no setor privado, dado encontrado em outros trabalhos ${ }^{9,12,28}$. Nota-se que a maioria dos egressos mudou de estado ou cidade, após a graduação, contrapondo a ideia de que o Tocantins apresenta oportunidade melhores e mais viáveis, relacionando a busca por um mercado de trabalho com maior oportunidade ${ }^{29}$.

Um resultado interessante deste estudo mostrou que um em cada 4 egressos optou pela atuação profissional em ambos os serviços, público e privado. Este fato pode ser interpretado como uma oportunidade para o recém-formado, que mesmo diante da crise atual no mercado de trabalho brasileiro, usa da garantia salarial do serviço público para honrar suas despesas fixas e apoia-se no salário extra oriundo do serviço privado ${ }^{30,31}$. Neste aspecto a Odontologia tem uma vertente de atuação autônoma, que pode ser exclusivamente privada ou através da inserção de profissionais em convênios, empresas, associações e sindicatos ${ }^{32}$.

Uma demanda importante avaliada neste estudo foi o anseio dos egressos ao término da graduação em relação à pósgraduação, onde a maioria já possuía ou estava cursando alguma pós-graduação, o que confirma a tendência da expansão da especialização observada nos últimos anos no Brasil $^{4,12,19}$ e a oportunidade de diferenciar-se, agregar qualidade e obter um melhor desempenho para competir no mercado de trabalho ${ }^{21,33}$. Concomitante a esta afirmativa observou-se que as áreas de Ortodontia (17,5\%), Endodontia (14,8\%) e Odontopediatria $(7,6 \%)$ foram as mais mencionadas dentre os egressos. Em contraponto, cursos como Odontologia para Pacientes com Necessidades Especiais e Odontologia Hospitalar foram citados apenas uma vez. Já os cursos relacionados à Saúde Pública ou Saúde Coletiva tiveram baixa participação nas respostas, fato também verificado em estudos sobre a mesma temática ${ }^{22,28}$.

As disciplinas mais citadas pelos egressos em relação às suas preferências, foram Cirurgia Oral, Endodontia, Dentística, Odontopediatria, Prótese, Saúde Coletiva e Ortodontia, respectivamente. Diferentemente destes resultados, há estudo que apresenta a Ortodontia como a disciplina favorita dos egressos $^{4}$. Curiosamente, a pós-graduação mais cursada entre os egressos foi a Ortodontia, o que abre precedente para inferirmos que aspectos financeiros podem estar associados à escolha da especialidade a ser cursada, uma vez que esta não coincidiu com a disciplina favorita durante a graduação.

A maioria dos profissionais possuía renda mensal de até 10 salários mínimos, sendo que $46,4 \%$ recebiam até 5 salários mínimos. A inserção dos profisssionais no mercado de trabalho foi em sua grande maioria imediata, mostrando a necessidade dos mesmos. Este fato é também justificado com a inclusão do cirurgião-dentista na Estratégia de Saúde da Família (ESF), a qual encontra-se em franca expansão ${ }^{9}$. Ao avaliarmos o nível de satisfação em relação aos rendimentos mensais, a maioria classificou como regular ou bom entretanto, quando comparada à remuneração do médico, cirurgião-dentista, enfermeiro e outros profissionais da saúde, o cirurgião-dentista apresenta o segundo melhor rendimento ${ }^{9}$. Por outro lado, rendimentos entre três e cinco salários mínimos foram considerados satisfatórios pelos cirurgiões-dentistas ${ }^{24}$. Outros estudos mostraram que os cirurgiõesdentistas se apresentam infelizes com a atuação profissional e queda do prestigio, e com isso acabam necessitando aumentar a carga horária de trabalho, dentre outros esforços ${ }^{15,34}$.

Vários estudos corroboraram com os resultados deste trabalho, uma vez que ocorre no Brasil, uma má distribuição dos 
profissionais nas regiões brasileiras ${ }^{9,21,22,35}$ contudo entende-se que a maioria destes opta por permanecer nas grandes cidades e nas áreas de melhores níveis de renda, a desbravar novas oportunidades e28,32,35 regiões, consequentemente aumentando a concentração e causado um acúmulo de ofertas de serviços ${ }^{9,21,22,29}$.

Este trabalho endossou a importância da IES realizar avaliações periódicas sobre a inserção de seus egressos no mercado de trabalho, com o objetivo de ponderar e julgar a formação do profissional ali desenvolvida, visando aproximar as práticas pedagógicas ao campo de trabalho.

\section{CONCLUSÃO}

O curso de Odontologia da FAPAC/ITPAC-PORTO tem formado profissionais com o perfil predominante jovem, do gênero feminino, naturais da região norte e nordeste do Brasil, que se inseriram no mercado de trabalho de forma rápida, obtendo renda mensal classificada como satisfatória. Além disso, notou-se uma tendência de atuação em consultório particular, atendendo uma população de renda média. Outro aspecto importante foi o fato da maioria dos egressos ter cursado ou estar cursando uma pósgraduação, sendo as mais citadas a Ortodontia e Endodontia.

\section{ABSTRACT}

Introduction of newly graduated dental students from the State of Tocantins into the job market.

The aim of this study was to investigate the insertion and professional perspective of dental graduates into the labor market. An observational, cross-sectional and descriptive study was performed, by means of an electronic questionnaire, to a sample of 298 Dentistry graduates from the State of Tocantins, Brazil. The sample showed 14 classroom groups from the years 2008 to 2015. The response rate was $61.41 \%(\mathrm{n}=$ 183). After data analysis (chi-square test /
Fisher's exact, 5\% significance level) it was found that $65.6 \%$ of respondents were female, mean age $30( \pm 6.6)$ years. The average time since their graduation was $3.7( \pm 2.2)$ years. Among the graduates, $37.8 \%$ are now working in Public Health, and $17.5 \%$ do so, exclusively. Most of them work in the State of Tocantins $(65.0 \%)$ and $62.8 \%$ have attended or are still attending post-graduation courses in Orthodontics (17.5\%), Endodontics $(14.8 \%)$, Pediatric Dentistry $(7.6 \%)$ and Public Health $(3.8 \%)$. The monthly income of the majority $(46.4 \%)$ is up to 5 times the minimum wage (R \$3,640.00) and $49.8 \%$ of the respondents reported a sense of achievement with the monthly income. The immediate insertion in the labor market was reported by $72.7 \%$ of the graduates, and $6 \%$ do not work as dental professionals, $2.7 \%$ of these have never done it. Among the most significant subjects while undergraduate students, Oral Surgery (26.8\%), Endodontics (21.3\%), Restorative Dentistry (14.2\%), Pediatric Dentistry (11.5\%), Prosthodontics (9.3\%) and Public Health $(4.9 \%)$ were the most meaningful for them. According to the results found it was concluded that the insertion of Dentistry graduates in the labor market was quick and the majority are happily satisfied with the income.

Descriptors: Job Market. Dental Staff. Staff Development.

\section{REFERÊNCIAS}

1. Brasil. Censo da educação superior. Ministério da Educação-INEP. Disponível em: download.inep.gov.br/ download/superior/censo/2013/resumo_t ecnico_censo_educacao_superior_2013. pdf

2. Morita MC, Kriger L. Mudanças nos cursos de Odontologia e a interação com o SUS. Rev ABENO. 2004;4(1),17-21.

3. Feuerwerker L, Almeida M. Diretrizes Curriculares e projetos pedagógicos: é tempo de ação! Rev ABENO. 2004; 4(1):14-7.

4. Brustolin J, Brustolin J, Toassi RFC, 
Kuhnen M. Perfil do acadêmico de Odontologia da Universidade do Planalto Catarinense - Lages - SC, Brasil. Rev ABENO. 2006; 6(1):14-7.

5. Conselho Federal de Odontologia. Profissão Cirurgião-Dentista: Uma profissão de muito trabalho em prol da população. [Acesso em: 30/04/2015]. Disponível em http://cfo.org.br/todas-asnoticias/17829/

6. Brasil. Brasil Sorridente completa 11 anos de atividade - UnaSUS. Ministério da Saúde; [Acesso em: 26/03/2015]. Disponível em: http://www.unasus.gov. br/noticia/brasil-sorridente-completa-11anos-de-atividade

7. Brasil.. Brasil tem maior número de dentistas no mundo, mas distribuição de profissionais é deficiente. [Acesso em: 10/10/2014]. Disponível em: http://www. dentistahoje.com.br/brasil-tem-maior-nu mero-de-dentistas-no-mundo-mas-distri buicao-de-profissionais-e-deficiente/

8. Pereira AC, Mialhe Fl, Pereira SM, Meneghim MC. O mercado de trabalho odontológico em saúde coletiva: possibilidades e discussões. Arqu Odontol. 2010; 46(4), 232-239.

9. Morita MC, Haddad AE, Araújo ME. Perfil atual e tendências do CirurgiãoDentista brasileiro. Dental Press Int. Maringá : PR. 2010.

10. Brasil. Política nacional de saúde bucal. Ministério da Saúde, 2004. [Acesso em: 28/04/2015]. Disponível em: http:// bvsms.saude.gov.br/bvs/publicacoes/poli tica_nacional_brasil_sorridente.pdf

11. Brasil. CNE. Resolução CNE/CES 3/2002 [internet]. Diário Oficial da União, Seção 1, p.10; Brasília, 4 de março de 2002 [Acesso em: 10/01/2015]. Disponível em: http://portal.mec.gov.br/ cne/arquivos/pdf/CES032002.pdf

12. Fadel CB, Baldani MH. Percepções de formandos do curso de Odontologia sobre as diretrizes curriculares nacionais. Trab Educ Saúde. 2013;11(2),339-54.

13. Araujo ME. Palavras e silêncios na educação superior em Odontologia. Ciênc Saúde Coletiva. 2006; 11(1),17982.

14. Mello ALSF, Moysés ST, Moysés SJ. A universidade promotora de saúde e as mudanças na formação profissional. Interface. 2010; 14(34), 683-92.

15. Ferreira NP, Ferreira AP, Freire MCM. Mercado de trabalho na Odontologia: contextualização e perspectiva. Rev Odontol UNESP. 2013; 42(4), 304-09.

16. Oliveira DL, Souza ES, Batista FJN, Alves JV, Yarid SD. Perfil do aluno de Odontologia da Universidade Estadual do Sudoeste da Bahia-UESB. Saúde Com. 2013; 9(3):169-78.

17. Cavalcanti YW, Lucena EHG, Wanzeler MC, Padilha WWN. Qualificando uma estratégia formadora: a proposta dos estágios da graduação em Odontologia da UFPB. Rev ICO. 2008; 6(2). [Acesso em: 10/01/2015]. Disponível em: http://periodicos.ufpb.br/ojs/index.php/re vico/article/view/3247/2710

18. Almeida AB, Alves MS, Leite ICG. Reflexões sobre os desafios da Odontologia no sistema único de saúde. Rev At Prim Saúde. 2010; 13(1):126-32.

19. Freire MCM, Jordão LMR, Ferreira NP, Nunes MF, Queiroz MG, Leles CR. Motivation towards career choice of Brazilian freshman students in a fifteenyear period. J Dent Educ. 2011 Jan;75(1):115-21.

20. Unfer B, Rigodanzo L, Hahn D, Manfredini D, Rodrigues E, Cavalheiro C. Expectativas dos acadêmicos de Odontologia quanto a formação e futura profissão. Rev Saúde 2004. 30(1-2):3340.

21. Mialhe FL, Furuses R, Gonçalos CS. Perfil profissional de uma amostra de egressos da Faculdade de Odontologia de Piracicaba. Rev Odontol UFES. 2008; 10(2):31-6.

22. Rezende FP, Nakanishi FC, Machado ACP, Quirino MRS. Perfil, motivação e expectativas dos graduandos e graduados 
em Odontologia. Rev Odontol UNICID. 2007; 19(2),165-72.

23. Costa SM, Durães SJA, Abreu MHNG. Feminização do curso de Odontologia da Universidade Estadual de Montes Claros. Ciênc Saúde Coletiva. 2010; 15(supl. 1), 1865-73.

24. Pinheiro VC, Menezes LMB, Aguiar ASW, Moura WVB, Almeida MEL, Pinheiro FMC. Inserção dos egressos do curso de Odontologia no mercado de trabalho. RGO. 2011; 59(2), 277-83.

25. Silva AC, Franco MM, Costa EL, Assunção HR, Costa JF. Perfil do acadêmico de Odontologia de uma universidade pública. Rev Pesq Saúde 2011. 12(1):22-6.

26. Toassi RFC, Davoglio RS, Lemos VMA. Integração ensino-serviço-comunidade: o estágio na atenção básica da graduação em Odontologia. Educ Rev 2012; 28(4): 223-42.

27. Santos KT, Filho ACP, Garbin CAS. Educação em saúde bucal na visão de acadêmicos de Odontologia. Arqu Bras Odontol Belo Horizonte. 2012; 48(2):96101.

28. Barbosa KGN, Dias JN, Cavalcante MS, Nobrega LM, Garcia AFG, Davila S. Formação e perspectiva do mercado de trabalho sob o olhar de alunos de Odontologia. Pesq Bras Odontoped Clin Int. 2013; 13(1):89-94.

29. Silva MAM, Amaral JHL, Senna MIB, Ferreira EF. O Pró-Saúde e o incentivo à inclusão de espaços diferenciados de aprendizagem nos cursos de Odontologia no Brasil. Interface. 2012; 16(42):707-17.
30. Junqueira JC, Colombo CLD, Tavares PG, Rocha RF, Carvalho YR, Rodrigues JR. Quem é e o que pensa o graduando de Odontologia. Rev Odontol UNESP. 2002; 31(2):269-84.

31. Rosing CK, Silva DT, Deon P, Oppermann RV, Gjermo P. Avaliação de 4 currículos de Odontologia baseada em expectativas e satisfação de alunos relato de experiências norueguesa e brasileira. Rev ABENO. 2009; 9(2):8894.

32. Garbin CAS, Garbin AJI, Santos KT, Hidalgo LRC, Moimaz SAS. Conhecimento sobre saúde bucal por concluintes de pedagogia. Rev Trab Educ Saúde. 2012;10(3):453-62.

33. Freitas CHSM. Conflicts in the practice of dentistry: the autonomy in question. Interface. 2007. 11(21):25-38.

34. Tiedmann CR, Linhares E, Silveira JLGC. Clínica integrada odontológica: perfil e expectativas dos usuários e alunos. Pesquisa Brasileira em Odontopediatria e Clínica Integrada, 2005;5(1):53-58.

35. Paranhos LC, Ricci ID, Filho RPA, Castro R, Scanavini MA, Análise do mercado de trabalho odontológico na região norte do Brasil. Rev Odontol São Bernardo do Campo-SP. 2009; 17(34):27-36.

\section{Correspondência para:}

Prof. Flávia Martão Flório

e-mail: flavia.florio@slmandic.edu.br

Rua José Rocha Junqueira, 13

13045-755, Campinas/SP 